\title{
Thinking about Ebola
}

\author{
${ }^{1}$ DM Brett-Major \\ ${ }^{1}$ Infectious Diseases Directorate, Naval Medical Research Center, Silver Spring, MD, USA; Division of Tropical Public Health, Department of \\ Preventive Medicine and Biometrics, F. Edward Hébert School of Medicine, Uniformed Services University, Bethesda, MD, USA
}

KEYWORDS Ebola virus disease, epidemic, infection prevention, outbreak

DECLARATION OF INTERESTS No conflict of interest declared

\author{
Correspondence to DM Brett-Major \\ Infectious Diseases Directorate \\ Naval Medical Research Center \\ 503 Robert Grant Avenue \\ Silver Spring \\ MD 20910 \\ USA
}

e-mail david.m.brett.mil@mail.mil
The current outbreak of Ebola virus disease (EVD) in West Africa involves members of the Royal College of Physicians of Edinburgh supporting the response on the ground, particularly in Sierra Leone, and I offer these thoughts as a clinician who had been transplanted into the outbreak at various times and locations from overseas. Those most experienced in the management of the outbreak are those residents of affected countries who continue to work to prevent, respond and contain the outbreak in the long term, with little resource and less recognition.

Time moves quickly in outbreaks but understanding develops more slowly. For decades since the first EVD outbreak in Africa in 1976, our predecessors learnt about the pathogen and its consequences in brief periods of time and with limited numbers of patients. The perspective has been from the vantage of those critically ill, late presenting patients from remote communities, detected because of the severe course of their illness.

No reader will be surprised at the idea that this EVD outbreak in West Africa is different.' It is large. It is diverse. It has had time to develop its character. Some feel that this character is a marked departure from our previous understanding of EVD outbreaks; and that the pathogen continues to evolve, possibly with clinical consequences. We have accumulated sufficient experience with enough patients and places to get a better sense of the disease. We also have a larger pool of clinicians with direct experience. Those few forebears in the field from previous outbreaks may not be surprised at all.

Clinically, before this outbreak, the common perception was that EVD patients bleed then die. Sometimes they do. Like the vast majority of known diseases, patients present across a spectrum of severity. The historical case fatality rate (CFR) of 9 out of 10 patients has regressed to a new mean, although we are not sure yet what that mean is. A publication by the World Health Organization (WHO) reviewing the first 9 months of the current outbreak asserted that West Africa experienced a CFR of nearly $71 \% .^{2}$ However, patients who present to care, especially early to care, are likely to fare better. Effective care centres in the right circumstances have achieved a CFR of less than $50 \%$ in affected areas. Referral centres outside of Africa have achieved very low CFRs with small numbers of patients.

Clinicians have come to appreciate more broadly that care of EVD patients can be both safe and effective. ${ }^{3}$ Many good infection prevention and control practices lead to more efficient, focused time in the isolation area directly tending to patients. Safe and effective care is practicable and important in resource limited as well as advanced care settings. Published reports of the details of presentation and care exist for some of the affected countries and exported cases. ${ }^{4-7}$

Clinical care is a core part of the overall outbreak response, not an activity apart. Early case identification, care of the patient and robust contact tracing and monitoring are inextricably linked and essential in the interruption of chains of transmission as well as part of the foundation of community/response team relationships. While broad community empowerment and behavioural change strategies are important in any outbreak setting, early containment of an outbreak requires a comprehensive clinical management strategy. This strategy must recognise the importance of safely achieving good clinical outcomes in EVD patients while reinforcing other elements of the response.

Broader clinical perspectives have been published. ${ }^{3}$ Several of the WHO clinical consultants in this outbreak have constructed key points around executing safe and effective care for suspected and confirmed EVD patients. ${ }^{8}$ There are book chapters on EVD. ${ }^{9}$ As to clinical preparedness, there are no substitutes for keeping up-to-date with relevant guidelines and recommendations., ${ }^{1,1,11}$

As a physician, I cannot help but focus on my own clinical experience. While I participated in patient care in varied places and times in the outbreak, such lessons are 
inherently biased. Each responding clinician experiences a different outbreak, as patients and communities are unique.

A two to four day non-distinct febrile prodrome was common. Occasionally a patient would present without fever but with other symptoms. This was rare and fever almost always developed soon after presentation. Malaria infection on presentation or subsequently during the hospital course also was common, diagnosed clinically or with specific DEFINE rapid assays. Patients with malaria and EVD seemed to have more challenging clinical courses. Distinct EVD clinical syndromes have been useful in approaching patients in my care, though the syndromes are not easily represented by the way that signs and symptoms typically are recorded in the outbreak.

Febrile arthralgia was demonstrated at presentation by a small group of patients with low mortality, 1:5-10 when admission registries were available for review. In overt clinical appearance these patients appeared similar to those with mild to moderate dengue fever. Adverse outcomes in this group were unusual, though occasionally a stroke or other isolated event occurred, particularly in those patients who were older and had comorbidities. Sometimes patients in this group would develop limited gastrointestinal symptoms. I have been suspicious that these patients were demonstrating a sero-conversion arthropathy. In some ways they reminded me of children with post-meningococcal arthritis. Patients who presented initially with febrile enteropathy sometimes develop this clinical picture during recovery.

Diarrhoea with or without vomiting was the dominant symptom in the febrile enteropathy group. Sometimes vomiting persisted but in other instances it was limited and sporadic. Fluid losses were high. Some patients, particularly those who had been symptomatic for a few days prior to presentation, had a 10 litre or greater water deficit and persistent 4-6 litre daily resuscitative requirements. Patient recognition of the importance of replenishing volume in the setting of diarrhoea at home was unusual. Sometimes I could rely upon oral rehydration solution intake, particularly when patients coached each other using intake targets. However, parenteral volume and empiric electrolyte repletion was often needed. Most patients improved or declined within a week of admission, but the clinical course could vary for up to three weeks and still result in survival.Variable non-focal abdominal pain, sometimes cramping and migratory with bloating similar to giardiasis, at other times briefly focal with rebound that soon afterwards was absent, was common. There seemed to be a slight epigastric and right upper quadrant preponderance to the discomfort.

Transient improvements of less than two days would occur, but full improvement for more than two days was not followed by recurrence of symptoms. In some instances, limited point-of-care chemistry information on patients was available. Profound metabolic acidosis was common, sometimes also with an overlying hyperchloremic metabolic acidosis depending on the resuscitative fluid availability. Hypokalaemia was common and sometimes profound. Many patients had a hepatocellular dominant hepatitis with transaminases of several hundred $U / L$. Amylase, lipase and coagulation studies were not available to me though others have reported that pancreatic enzyme elevations were also common. Cytologic analysis was not reliable in my settings.

Moderate to severely ill patients often developed a deep central chest discomfort and anxiety that persisted and was difficult to manage either because of a lack of more potent analgesia or inability to visit frequently enough to administer short acting formulations. Thrush was common. A wide range of mental status changes were apparent in this group. Hiccups often seemed to be associated with declining volume status or worsening acidosis and would resolve with volume repletion over hours to days, returning when there was a drop in fluid replacement. It was a clinical warning that triggered escalations in fluid replacement similar to increasing compensatory tachypnea from metabolic acidosis. This is in contradistinction to others' experience in previous outbreaks where hiccups had been perceived to be a pre-terminal sign. Some patients had non-pitting oedema during resuscitation but had persistent intravascular volume deficits that were apparent through measuring orthostatic pulse and blood pressure.

Bleeding in general was a late sign and then potentially profound; not, however, mild to moderate mucosal bleeding (oropharyngeal, melaena, haematochezia) sometimes occurred early during presentation and then resolved in recovering patients.

Most references discuss a rash but, in my experience, the rash was variably present. It was a fine, mostly central papular rash that was sometimes pruritic. Patients rarely mentioned the rash without prompting. I usually noted it while palpating and not on casual inspection. The rash reminded me of presentations of secondary syphilis and vasculitis. A test for syphilis on Ebola infected blood was not available to me.

Oxygen requirements were rare. When oxygen requirements were present, the patient appeared to have a pulmonary embolus or pneumonia. Tachypnoea was common. In patients without an apparent or likely pulmonary event, they had no insight into their respiratory effort. The tachypnoea appeared compensatory for the patients' metabolic acidosis.

Confusion, behavioral disturbance and the 'starry-eyed stare' were common findings among moderately and severely ill patients. Metabolic derangement either from electrolyte loss, renal or hepatic injury, viral effects, 
consequences of uncontrolled pain, adrenal insufficiency and acute nutritional deficits all seemed possible in various patients. Seizures occurred but were uncommon. In some patients, who appeared at risk for vasculopathy (obesity especially with adult-onset diabetes and older age), cognitive deficits were more marked. Occasionally, paratonia, perseveration and frank delirium were apparent. This last group of patients was particularly difficult to manage and seemed to have a higher incidence of what could have been cerebrovascular events with stepwise deterioration, although severe metabolic disturbances could not be excluded in some settings. Mortality was high. While gastrointestinal signs and symptoms were present in this group, the neurocognitive features were more persistent.

Increasing experience with patients in referral critical care units has been gained in a wide range of centres, including at the Royal Free Hospital in London as well as in Germany, Italy, Spain, France and across the United States. Patient presentation and care in these settings has distinct features in contrast to field settings where the patients are from the local community and full volume repletion and advanced interventions are unlikely. Lung injury and other consequences of later stage disease as well as volume overload were sometimes observed in the course of aggressive, successful care.

Managing these patients in any setting is like managing any patient with severe sepsis or shock, using targeted strategies appropriate to the patient's needs and the level of resources available. The broad, direct effects of the virus, volume and electrolytes status, the presence of concomitant or supervening infections, organ injury, delirium, co-morbidities including nutritional status, palliation of sometimes profound pain and anxiety and basic elements of patient safety when hospitalised or isolated all require attention. Robust infection prevention and control practice must be integral to how this is done.

\section{REFERENCES}

I World Health Organization (WHO). Ebola Virus Disease (EVD). http://www.who.int/csr/disease/ebola/en/ (accessed 28/10/20l4).

2 WHO Ebola Response Team. Ebola virus disease in West Africa - the first 9 months of the epidemic and forward projections. New Engl J Med 20I4; 37I: |48|-95. http://dx.doi.org//0.1056| NEJMoal 4 III 100

3 Fowler RA, Fletcher T, Fischer WA 2nd et al. Caring for critically ill patients with ebola virus disease. Perspectives from West Africa. Am J Respir Crit Care Med 2014; 190: 733-7. http://dx.doi. org/I0.1I64/rccm.20I408-15I4CP

4 Bah El, Lamah MC, Fletcher T et al. Clinical presentation of patients with Ebola virus disease in Conakry, Guinea. N Engl J Med 2015; 372: 40-7. http://dx.doi.org//0.1056/NEJMoa I4I/249

5 Schieffelin JS, Shaffer JG, Goba A et al. Clinical illness and outcomes in patients with Ebola in Sierra Leone. N Engl J Med 2014; 37I: 2092-100. http://dx.doi.org/I0.1056/NEJMoa I4II680
Looking ahead, the biodefence industry has brought several diagnostic, preventive and therapeutic products in use or near the point of beginning clinical assessment. WHO facilitates and reports on these through its New Interventions initiative.' The most critical intervention is the introduction of clinical practice which we all would expect at our own centres. Should EVD therapeutics be validated and more available, they may be useful for a range of patient-centred outcomes. Decreasing mortality is important as is the reduction of time spent in isolation, making patients feel better, preventing disease in close contacts of confirmed cases, deconstructing the barriers to receiving critical non-EVD care and re-launching broad public programming around managing maternal haemorrhage, childhood respiratory and enteric disease reduction and treatment, malaria, HIV, TB and many others. Improvements in the management of pregnant EVD patients, very young children and those with neuropredominant syndromes would be welcome.

The gains in this outbreak have been slow, hard won and sometimes transient. Hopefully, we will see those gains include more baseline capacity that endures and increased preparedness for the next special pathogens outbreak.

\section{Disclaimer}

The views expressed in this article are those of the author and do not necessarily reflect the official policy or position of the Uniformed Services University of the Health Sciences, Department of the Navy, Department of Defense, nor the US Government.

DMB is a military service member. This work was prepared as part of his official duties. Title 17 USC section 105 provides that 'Copyright protection under this article is not available for any work of the US Government.' Title I7 USC section I0I defines a US Government work as a work prepared by a military service member of the US Government as part of that person's official duties.
6 Lyon GM, Mehta AK, Varkey JB et al. Clinical care of two patients with Ebola virus disease in the United States. N Engl J Med 2014; 37I: 2402-9. http://dx.doi.org/I0.I056/NEJMoal 409838

7 Kreuels B, Wichmann D, Emmerich P et al.A case of severe Ebola virus infection complicated by gram-negative septicemia. N Engl J Med 20I4; 37I: 2394-40I. http://dx.doi.org/I0.1056/ NEJMoa I4II677

8 Brett-Major DM, Jacob ST, Jacquerioz FA, et al. Being ready to treat Ebola virus disease patients. Am J Trop Med Hyg 2015; 92: 233-7. http://dx.doi.org// 0.4269/ajtmh. I4-0746

9 Peters CJ. Ebola and Marburg Viruses. In: Longo DL, Fauci AS, Kasper DL et al., editors. Harrison's Principles of Internal Medicine. 18th ed. New York, NY: McGraw-Hill: 2012. p. 1633-8.

10 Centers for Disease Control and Prevention. Ebola (Ebola Virus Disease). http://www.cdc.gov/vhf/ebola/index.html (accessed 28/10/2014).

II Médicins Sans Frontières (MSF). MSF Reference Books. http:// refbooks.msf.org/msf_docs/en/MSFdocMenu_en.htm (accessed 28/10/2014). 